\title{
The Verification Analysis of the Software Dynamic Evolution Topology Structure Model Based on Demand and Runtime Variability Parallel Driver under the Background of Large Data
}

\author{
Liu Zhigang ${ }^{1,2}$, Li Tong ${ }^{1}$, Yu Xiaonan ${ }^{2}$, Wang Xiaozhi ${ }^{* 2, a}$ \\ ${ }^{1}$ National Pilot School of Software, Yunnan University, Kunming 650500,China; \\ ${ }^{2}$ Nanchang Institute of Science \&Technology,Nanchang 330108,China \\ apattywxz@126.com
}

\begin{abstract}
Keywords: Dynamic evolution, Requirement change, SaaS software, Communication process algebra, EPMM modeling.
\end{abstract}

\begin{abstract}
Due to consider the deficiency of the dynamic software evolution in the current demand engineering stage, this paper goes through the change demand forecasting and the sensitivity analysis based on the relationship between software runtime variability and change process, to establish the double drive model for SaaS software dynamic evolution. To solve the verification problem of facing the dynamic evolution, this paper is the establishment of supporting theoretical basis and verification method, to produce the software dynamic evolution process of EPMM modeling. From the perspective of process algebra, this paper describes the software process, but also the use of communication process algebra (ACP) extends on EPMM. Through more scientific decision-making improvement software process, we can obtain better software quality, faster development evolution efficiency, better risk control and more revenue. Using the sensitivity analysis results, we can determine whether to adopt the software process change, and change sensitivity analysis results can be as the software process of organization planning, improvement implementation and improvement tracking, which does beneficial supplement for the theoretical study of the existing requirements engineering.
\end{abstract}

\section{Introduction}

This paper, for the first time, put forward the concept of software engineering at the 1968 NATO Conference, and then it proposed to the software development from the art and individual behavior to project and group cooperative work transformation [1-3]. System software size is larger and larger, the complex degree is higher, software reliability problem is more and more outstanding, the demand of software system and software complexity is also rising rapidly, because the traditional development methods are unable to adapt to the software needs of users in terms of quality and efficiency, so we urgent need to change the mode of software production, to improve software productivity [4,5]. In order to solve this problem, this paper applies the computer science theory and technology as well as project management principles and methods, to meet the user requirements' software product definition, development, deployment and engineering maintenance in accordance with the budget and schedule [6-8]. After decades of continuous efforts, the theory of software engineering has been greatly enriched and perfect, all kinds of software design methods are constantly emerging, software industry is booming, so as to promote the continuous development of computer science.

Internet speeds up the information to enter all aspects of the social economy and the daily life of the masses, especially the development of mobile internet. Data shows that the total amount of the Baidu Inc currently is close to $1000 \mathrm{~PB}$, the number of pages stored is close to 1 trillion pages, it deal with about 6 billion search requests every day, and there are dozens of PB data; hospital is also a place where data is generated, now the total amount of a patient's CT image data reach the dozens of GB, while the number of people in the country each year reach hundreds of billions of dollars, and their information needs to be stored for a long time [9]. On the one hand, the emergence and rapid development of cloud computing makes more and more successful software systems become a legacy 
system, and the importance and popularization of software evolution has become more and more strong; on the other hand, the software evolution environment starts from gradually distributed to centralize. How to effectively support the evolution of software systems in the open, dynamic, changeable and centralized cloud computing environment is an important challenge for software engineering.

\section{Existing Challenges and Opportunities in the Process of Software Dynamic Evolution Problems}

In the era of big data, there exist challenges and opportunities in the process of software dynamic evolution problems. In software dynamic evolution problems, academic circles carry out exploration and research from the following several aspects, including the software system structure, software platform, model and program design language [10-12]. Combined with the analysis of some existing results, we can found in some of existing successful and countermeasures as follows:

(1) Programming language angle. Researchers hope that they can through the design of high-level programming language to directly support dynamic software evolution, but in programming language, the dynamic evolution is lack of special theory guidance. So the intelligent will study limitations in the process, objects, functions and methods and so on.

(2) Model angle. Researchers have proposed a variety of system structure model, the typical of abstract algebra, ontology, conditional hypergraph and Agent method construct the software dynamic evolution system model, the models have good form, so it can better describe the evolution process. At the same time, the model can analyze the ripple effect of evolution and consistency, to analyze the dynamic evolution from the macro level. But these models are difficult to study and are lack of effective support means, so they often fall into the difficult position.

(3) The perspective of software architecture. Researchers establish software system model or system description language to describe the dynamic evolution, and some scholars pioneering use the software system structure to describe the dynamic evolution model, but they do not consider demand driven software evolution in essentially, they only study the system structure and the layer, they are often unable to adapt to the speed and flexibility of the changing requirements, leading to the requirements and architecture do not build mapping mechanism to deal with the dynamic evolution.

(4) Platform angle. Researchers hope to build a dynamic evolution platform, but so far, this condition is not mature yet. So under the background of big data, people currently face the problems and challenges constructed by software dynamic evolution platform, and the existing research results still exist much shortage and further improve the space.

\section{Research on the Dynamic Evolution of Double Driven Software Based on Demand and Operation under the Background of the Big Data}

For the collection and processing swarm intelligence data and software process duration data, it carries out the analysis and forecast for software requirement change, it are multi-source, heterogeneous, complex data analysis and other problems, so this paper needs to solve the problem of the big data analysis.

Dig data analysis. The combination of swarm intelligence data and software process diachronic data will appear big data, but also will be the formation of complex data sets, software technology and software engineering date. This paper researches complex data analysis methods, which can implement requirements and time-varying driven data application.

The dynamic evolution model of demand and variability double drive. How to support the software dynamic evolution in the cloud environment, the internal and external manifestations of software process are studied through the division of calculation model and structural model, to set up the double actuated model.

Supporting for dynamic evolution in the context of the big data. Under the background of large data, PaaS platform will gradually replace the traditional software development and operation platform. This paper puts forward the management mechanism of the cloud evolution, and it supports 
the software dynamic evolution under the environment of large data, which provide theoretical support for the realization of the future PAAS platform.

The reliability verification of dynamic evolution. The reliability verification of dynamic evolution includes behavior correlation verification and consistency validation. People need to carry out correlation analysis before the real-time dynamic evolution to avoid the process of dynamic evolution, because the implementation of target components affects the other components, and there are even serious mistakes. Considering the consistency of the analysis, it is proved that the analysis of the two problems is an important guarantee to verify the reliability of problem analysis after the implementation of the dynamic evolution, but also is an important guarantee to verify the internal state and components as well as the consistency of the external interaction.

The effectiveness of software process improvement. The ultimate goal of software process improvement is high efficiency, high quality and low cost production software, software process improvement focus is improving the effect, so this paper studies how to establish process improvement initiatives and to determine the improvement target, and finally this paper puts forward the improvement evaluation methods and update the model, to get effective software process improvement model.

\section{The Verification Analysis of the Dynamic Evolution based on Demand and Operation Double Driven Software under the Background of Big Data}

Due to ongoing changes of the demand in the open environment, demand software system has higher flexibility and scalability, so in many cases, the demand s of system software can be passive adjustment with the location changes and change extent, namely it requires the software can be dynamic evolution. When the software project and software organization are in a dynamic environment, the technology innovation, market changes and personnel flow will force software along with dynamic changes. In order to enhance the management and change of software process, this paper is the domestic relationship with variability and change based on the software, to establish the double actuated model of the oriented SaaS software dynamic evolution through the forecast and the sensitivity analysis of demand changes. And then, using the cross application of Petri nets and ACP supports the validation of software structure, properties and behavior.

Requirement change forecast and change sensitivity analysis. (1) Demand change forecast. In the entire cycle of software, there are a large number of historical data available. For the analysis of the historical data, it can provide the basis for the prediction of the demand change, and the accuracy of the analysis can be obtained by using the forecast data analysis. With the popularity of the network, a large number of software developers participate in the software development process in different regions, forming a large group of individuals. The wisdom of the group has made the prediction more accurate, but also has better dynamic and completeness. This paper goes through the historical process data and the group wisdom knowledge on the prediction of demand change, different knowledge will have effectively combined with a lot of information individual computer software engineering, so we can access to more dynamic and accurate changes in demand forecast results.

(2) Demand sensitivity analysis. After requirement change impact is through change propagation from a way or some way change, the ultimate impacts demand, so the estimation of demand comes from the sensitivity analysis of the external influence, and change impact analysis can be queried by recording. There are very strong dependence between the records and change, between the change and source change and between change and path process, using statistical will these relationships to carry on visualization and the establish a unified control channel, so as to determine whether to adopt the decision of the changes by the final results of sensitivity analysis. The sensitivity analysis results can be used as the basis of software process organization plan, improvement implementation and improvement tracking.

Establishing software dynamic evolution demand and time variable double driving model based on the oriented SaaS. Under the background of big data, cloud computing environment makes SaaS gradually become the mainstream form software, SaaS emphasizes the external manifestation of the software entity, while the inner is still a component, object and process, taking 
map as guidelines can ensure two types of traceability. In the external demand driven, user demand plays an important role in the life cycle of SaaS, and the user goes through structural model to understand and use the software, and user puts forward specific evolution intent for the software dynamics, so the structure model is a view of dynamic evolution analysis and implementation. In order to further support the effectiveness of dynamic software evolution, the establishment of the model needs to reflect the software operation, for example, the model can distinguish between stable and unstable components, the distinction between active and passive components, internal calculation for the components and relative isolation and other mechanisms. Therefore, it is necessary to establish support validation of the theoretical foundation.

Establishing the theoretical basis and verification method of supporting behavior. (1) To establish the support behavior theory. In actual software process, it involves many factors. The process model produced by the use of specific methods cannot advance a description of all the process details for a particular software project, so the software process model carries out verification, the model can be determined in line with our expectations, improving the reliable of software process implementation.

This paper produces dynamic software evolution process for EPMM modeling. From the perspective of process algebra, we carry out the software process description, and the use of the communication process algebra (ACP) expand the EPMM, as the derived form of the software process evolution verification mainly studies the validity and reliability of the research system.

In order to avoid the evolution process based on Petri nets and the grammar difference between process specifications based on ACP, EPMM is the algebra semantic domain of the evolution process, from the angle of semantic forms. Under the premise of behavioral equivalence, using the method of algebraic definition is derived based on the equation in the support of EPMM system axioms, so as to verify of software evolution behavior.

(2) Supporting behavior verification method. On the basis of the establishment of the verification theory, this paper will be applied to the Petri network and ACP. Combined with the advantages of the two, this paper puts forward the process algebra verification scheme based on Petri nets, and this scheme supports the model structure, properties and behavior verification of software evolution process. Using Petri net can be reachability graph intuitive specification and verification software evolution process, using ACP theory can be equation derivation and verification software evolution process under the support of EPMM system, so the content of this part mainly includes the following content:

(1) Software evolution process structure verification. The use of the relevant technology based on Petri net limits on the process model constraints, and designs the related checking algorithm to support process model validation.

(2) The property verification and behavior verification of supporting software evolution process model. Using Petri net related technology can be verified from the security and software development. In the EPMM system support, the use of equation derivation can verify the process equivalence.

(3) Improving the efficiency of verification. The model is simplified, as far as possible to reduce the number of model nodes, to alleviate the state space is facing the state explosion problem, which can improve the efficiency of the verification.

Improvement software process. Software process improvement includes two kinds of modeling method, in which the top-down approach takes software improved model as the theoretical basis, to carry on real-time software improvement based on the mature CMMI mode; the bottom-up method generally uses the theory-evaluation-packaged software process improvement method. This paper uses PAAS software dynamic evolution platform based on requirement changes and sensitivity demand forecasting, to improve software process under the background of big data. Finally, the use of Petri net and ACP verify the improvement process, to carry on evaluation and packaged for the improvement result. 


\section{Summary}

In order to solve the operating environment of the dynamic evolution, this paper builds the internal reflection of the SaaS evolution model, to describe the needs and variability of software dynamic evolution, the model uses the communication process algebra (ACP) as the main form, and each piece uses ACP to describe a process item. On this basis, based on cloud computing dynamic environment, this paper studies effective prediction software requirements and change sensitivity method in software process, and taking the requirements and operational variability for dual drive model establishes PAAS software dynamic evolution platform. Finally, this paper carries out improvement for the software process, the use of the Petri net and ACP verify the improvement process, and the improvement results are evaluated and packaged.

\section{Acknowledgments}

The work was supported by the Post Doctoral Orientation Funding Project of Yunnan Province in 2015. The work was also supported by the Project of Youth Science Foundation of National Natural Science Foundation with the project number 41505015 and the project name Measurement Method of Atmospheric High-resolution Microwave Radiometric Based on Virtual Mirror Antenna. Finally,the work was also supported by The science and technology research project of Education Department of Jiangxi Province in 2015 with the project number GJJ150156 and the project name Prediction of Forest Fire Spread Based on Grey GM $(1,1)$ Model.

\section{References}

[1] S. Wang. Research on software system dynamic evolution based on component. Yunnan University Software Institute, 2014(12): 1-13.

[2] R. Fan, Y.Q. Peng, Y.Q. Chen et al. A formal method of adaptive software based on extension theory. Journal of intelligent systems, 2015, 10(6): 901-911.

[3] H. Zhou, X.M. Wang, Z.M. Cai. A dynamic evolution framework of MPI parallel software. Computer application and software, 2016, 33(1): 8-10.

[4] Y. Zhou, Y.K. Huang, Z.Q. Huang et al. A kind of open environment software online evolution consistency verification method. Journal of software, 2015, 26(4): 749 -759.

[5] T. Li. An Approach to Modeling Software Evolution Processes. Springer-Verlag, Berlin, 2014(23): 1102-1112.

[6] Lehman M M. Laws of software evolution revisited. Proceeding of the European Workshop on Software Process Technology. Nancy, 2014(1): 108-124.

[7] C.Y. Li, P.J. He, et al. Software dynamic evolution technology. Peking University press, 2014: 132-140.

[8] Godfrey M W, German D M. The past present and future of software evolution. Frontiers of Software Maintenance, 2015(3): 670-678..

[9] H. Yang, M. Ward, Successful Evolution of Software System, Artech House, London, 2013(1): 112-116.

[10] J.W. Liu, X.J. Mao. Research on software runtime variability dynamic evolution mechanism. Journal of computer, 2015, 38(131): 1-18.

[11] C.Y. Li. Research on software dynamic evolution based on system architecture. Zhejiang University School of computer science and technology, 2015: 1-10.

[12] Lehman M M., Ramil J F, Wernick P D, et al. Metrics and laws of software evolution: The nineties view. Proceeding of the 4Intl. Software Metrics Symposium, Albuquerque, NM, 2014(11): 456-462. 